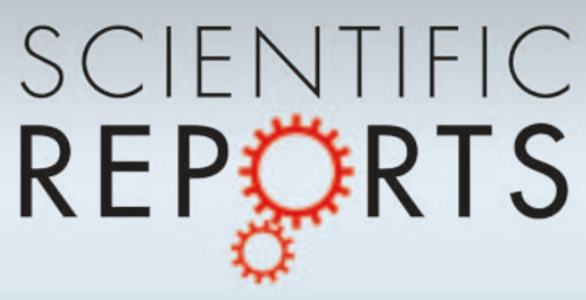

OPEN

SUBJECT AREAS:

APPLIED PHYSICS

CONDENSED MATTER PHYSICS

ELECTRONIC MATERIALS AND

DEVICES

SENSORS

Received

4 February 2012

Accepted

8 March 2012

Published

23 March 2012

Correspondence and requests for materials should be addressed to J.Q.L. (liq@aphy.iphy. ac.cn)

\section{Extreme chemical sensitivity of nonlinear conductivity in charge-ordered $\mathrm{LuFe}_{2} \mathrm{O}_{4}$}

\author{
Shi Cao, Jun Li, Zhen Wang, Huanfang Tian, Yuanbin Qin, Lunjie Zeng, Chao Ma, Huaixin Yang \& Jianqi Li
}

Beijing National Laboratory for Condensed Matter Physics, Institute of Physics, Chinese Academy of Sciences, Beijing 100190, P. R. China.

Nonlinear transport behaviors are crucial for applications in electronic technology. At the nonlinear critical turning point, the nonequilibrium states cause rich physics responses to environment. The corresponding study in this field is crucial for physics and industry application. Here nonlinear conductivity in charge-ordered (CO) $\mathrm{LuFe}_{2} \mathrm{O}_{4}$ has been demonstrated. Remarkable resistivity switching behavior was observed and the gas-sensing property can be reversibly tuned by a small alternation of partial pressure and/ or chemical components of the environment. These facts allow us to use $\mathrm{LuFe}_{2} \mathrm{O}_{4}$ materials as a sensitive chemical gas sensor in technological applications. Careful analysis of the gas sensing process in $\mathrm{LuFe}_{2} \mathrm{O}_{4}$ suggests a novel sensing mechanism in sharp contrast with that discussed for the conventional gas sensors which depend fundamentally on surface chemical reactions.

$\mathrm{n}$ past decades, the precise detections of environmental gas components have gained great importance in a variety of fields, including those of combustion control, food preservation, instrumentation of experiments, biology, medical engineering, and industry ${ }^{1}$. Conductometric sensor have been investigated intensively recently due to their simplicity, low cost, and small $\operatorname{size}^{2,3}$. On the other hand, the nonlinear current-voltage (I-V) character and switching of resistivity states in charge-ordered (CO) systems have been found under a variety of environmental conditions, such as magnetic field ${ }^{4}$, stress field ${ }^{5}$, electric field ${ }^{6}$ and with irradiation by $\mathrm{X}$ rays $^{7}$, respectively. We herein report our discovery of the remarkable gas sensitivity of resistivity in the charge/ spin frustrated $\mathrm{LuFe}_{2} \mathrm{O}_{4}$ which shows a remarkable charge-stripe order at room temperature ${ }^{8}$. Our experimental measurements of $\mathrm{LuFe}_{2} \mathrm{O}_{4}$ in a gaseous environment of $\mathrm{O}_{2}, \mathrm{He}, \mathrm{Ar}$ and vacuum often give rise to visibly distinctive transport features. In particular, remarkable switching behaviors of the conductivity are found above the threshold values in association with the field-driven $\mathrm{CO}$ melting or collective motions of charge stripes. This gas-sensing property can be reversibly tuned by a small alternation of partial pressure and/or chemical components of the environment. These facts demonstrate that the $\mathrm{CO} \mathrm{LuFe} \mathrm{O}_{4}$ materials could be used as chemical gas sensors with a fast response and a substantially higher sensitivity at room temperature. In sharp contrast with the conventional conductometric semiconducting gas sensors which depend on reversible interactions of the environmental gas with the surface of the materials ${ }^{9,10}$, the gas sensitivity of $\mathrm{LuFe}_{2} \mathrm{O}_{4}$ is found to be in correlation with the nonlinear conductivity influenced by the environmental gases.

\section{Results}

In order to characterize the essential transport nonlinearity in the layered $\mathrm{LuFe}_{2} \mathrm{O}_{4}$ crystal, we first performed our resistivity measurements on a number of single crystal samples along the relevant directions. Figures $1 \mathrm{a}$ and $1 \mathrm{~b}$ show the current density-voltage field (J-E) curves obtained from a single crystalline $\mathrm{LuFe}_{2} \mathrm{O}_{4}$ sample for the current flowing respectively parallel (Elc) and perpendicular (E// ab plane) to the c-axis direction, illustrating the presence of visible nonlinear J-E characteristics along the $c$-axis and within the ab plane in a $\mathrm{LuFe}_{2} \mathrm{O}_{4}$ crystal. The nonlinear behavior in each measurement can be clearly recognizable as a clear anomaly cross the threshold electric field $\left(E_{t}\right)$, the $E_{t}$ in general appear at around $60 \mathrm{~V} / \mathrm{cm}$ and $10 \mathrm{~V} / \mathrm{cm}$ for E parallel and perpendicular to the c-axis direction. According to the empirical power-law relation of $I=k V^{\alpha}$, the nonlinear coefficients of the single crystal can be estimated to be about 200 and 30 for the I//c and I $\perp$ c directions, these coefficients depend moderately on the current density used in transport measurements.

It has been commonly noted in previous investigations that visible alternation of resistance state could be triggered in a number of the $\mathrm{CO}$ systems $\mathrm{s}^{4-7}$. Our recent experimental investigations reveal that the nonlinear conductivity in association with $\mathrm{CO}$ melting in $\mathrm{LuFe}_{2} \mathrm{O}_{4}$ is extremely sensitive to the environmental conditions. Figure 2a shows nonlinear J-E behaviors taken from a well-characterized polycrystalline samples upon exposure 


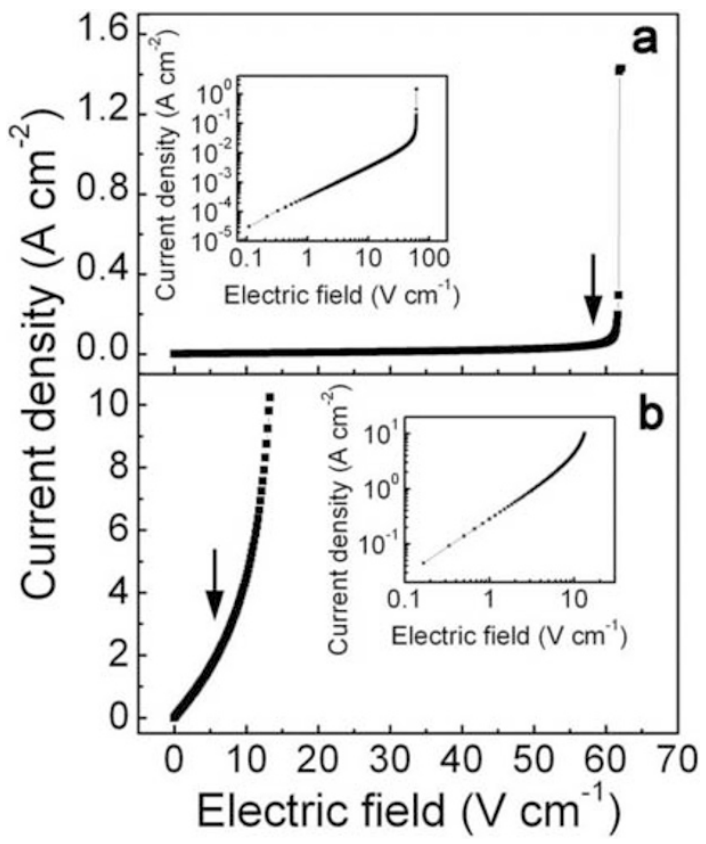

Figure $1 \mid$ Current density-electric field (J-E) curves of $\mathrm{LuFe}_{2} \mathrm{O}_{4}$ measured at room temperature: (a) nonlinear characteristics of the single-crystalline $\mathrm{LuFe}_{2} \mathrm{O}_{4}$ with the applied electric field parallel to the caxis; (b) J-E curve of the single crystal with electric field perpendicular to the c-axis. The arrows denote the threshold electric fields for nonlinear conduction. In the insets of $(\mathrm{a}, \mathrm{b})$ the log-log plots obtained numerically from the corresponding J-E curves are also shown.

to different gaseous environments. It is recognizable that a jump-like transition of electric current yields a visible nonlinear conductivity at a threshold electric field, or corresponding threshold currents for each curve. The most striking phenomenon revealed in our measurements is the notable divergent behaviors above the threshold, demonstrating the existence of atmosphere-dependent J-E characteristics in the $\mathrm{CO} \mathrm{LuFe}_{2} \mathrm{O}_{4}$ material. It is also noted that the sample in a $\mathrm{He}$ atmosphere shows a relatively high threshold current and a larger resistivity in comparison with the data obtained in $\mathrm{O}_{2}$ and Ar atmosphere. According to the results shown in Fig. 2a, we can estimate the threshold current values for $\mathrm{LuFe}_{2} \mathrm{O}_{4}$ under different environmental conditions as: $\mathrm{J}=0.5 \mathrm{~A} / \mathrm{cm}^{2}$ for vacuum $\left(\sim 10^{-1} \mathrm{~Pa}\right), 0.7 \mathrm{~A} / \mathrm{cm}^{2}$ for $\mathrm{O}_{2}$, and $0.9 \mathrm{~A} / \mathrm{cm}^{2}$ for $\mathrm{He}$, respectively. Moreover, it is also noted that the gas sensitivity depends strongly on applied current in experimental measurements, i.e. the larger electric current is used, and the bigger difference appears in data of the resistivity. This feature is also recognizable in Fig. $2 b$ which shows a series of resistivity curves under different measuring currents above threshold value from $0.3 \mathrm{~A} / \mathrm{cm}^{2}$ to $3.0 \mathrm{~A} / \mathrm{cm}^{2}$. This measurement was first performed on a $\mathrm{LuFe}_{2} \mathrm{O}_{4}$ sample under ambient conditions, and then the $\mathrm{O}_{2}$ was pumped out to lower the chamber pressure progressively to $10^{-1} \mathrm{~Pa}$. The remarkable effects of electrically driven non-linear resistivity and visible resistivity changes with alterations of the $\mathrm{O}_{2}$ partial pressure are plainly illustrated.

Though there is no uniform definition for the gas-sensor sensitivity now, we can use the ratio of $S_{R}=\frac{R_{o x y}-R_{\text {vac }}}{R_{o x y}} \times 100 \%$ to qualitatively illustrate the sensitivity in our measurements. According our experimental data shown in Fig. $2 \mathrm{~b}, \mathrm{~S}_{\mathrm{R}}$ depends strongly on the applied electric current, for instance, it is estimated to be $\sim 37.8 \%$ and $72.7 \%$ for $\mathrm{J}=0.3 \mathrm{~A} / \mathrm{cm}^{2}$ and $3.0 \mathrm{~A} / \mathrm{cm}^{2}$, respectively.

In order to understand the gas sensitivity in correlation with the charge order in $\mathrm{LuFe}_{2} \mathrm{O}_{4}$, we have checked the temperature dependence of the resistivity switching in the temperature range from $295 \mathrm{~K}$ a

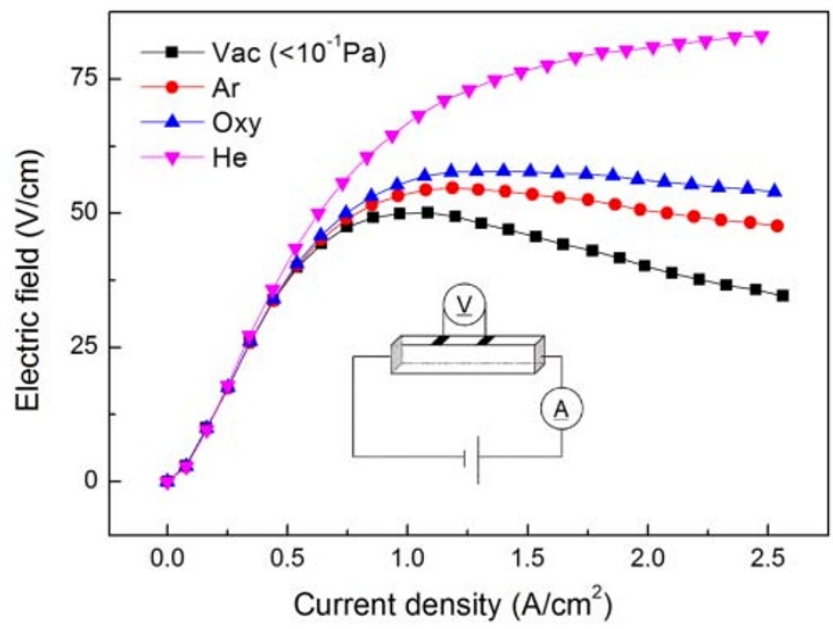

b

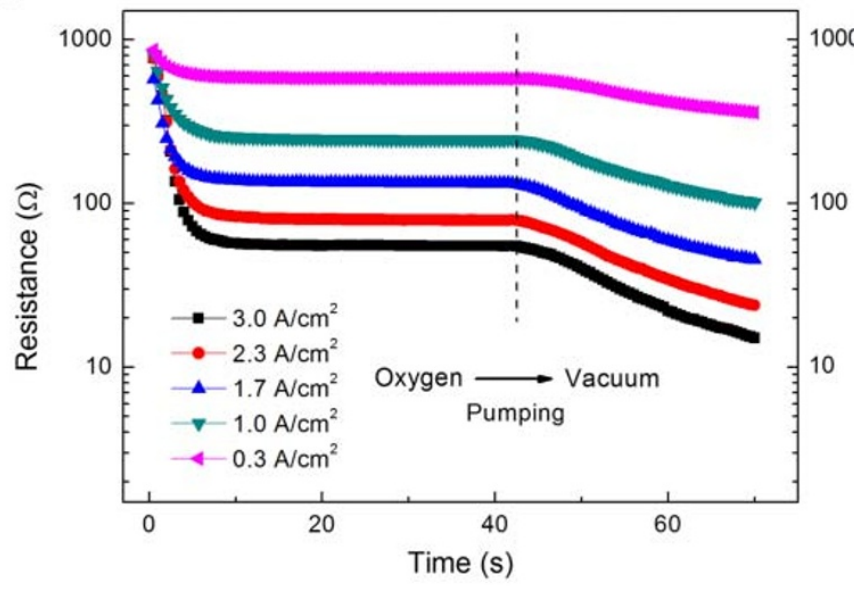

C

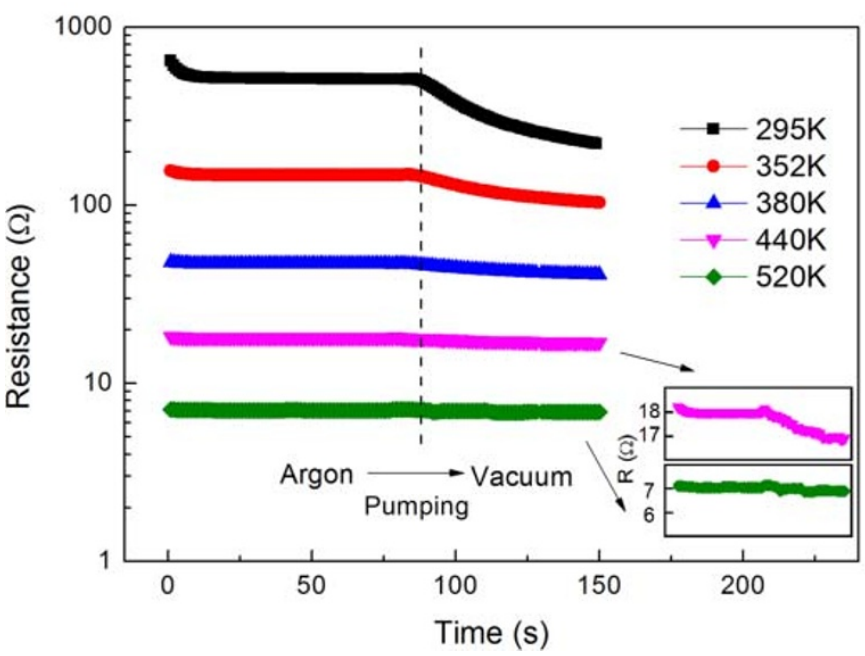

Figure $2 \mid$ Resistance switching character of $\mathrm{LuFe}_{2} \mathrm{O}_{4}$ at roomtemperature. (a) Nonlinear current density-voltage field (J-E) behaviors at different atmosphere. Pulsed voltages with the width $200 \mathrm{~ms}$ and interval $600 \mathrm{~ms}$ were used. Vacuum of about 0.1 Pa was obtained by a mechanical pump. (b) A series of resistance curves under different testing current from $0.3 \mathrm{~A} / \mathrm{cm}^{2}$ to $3.0 \mathrm{~A} / \mathrm{cm}^{2}$. Current is continuously applied on sample by four probe methods for resistance testing. (c) Resistance curves at different temperatures from $295 \mathrm{~K}$ to $520 \mathrm{~K}$ between vacuum and argon under testing current $0.3 \mathrm{~A} / \mathrm{cm}^{2}$. Inset shows details of $\mathrm{R}(\mathrm{T})$ curve at $440 \mathrm{~K}$ and $520 \mathrm{~K}$ respectively. 
to $520 \mathrm{~K}$ and in the gaseous environment of $\mathrm{Ar} / \mathrm{vacuum}$ in which the $\mathrm{LuFe}_{2} \mathrm{O}_{4}$ sample can be safely heated to high temperatures above the charge ordering transition temperature $\left(\mathrm{T}_{\text {co }} \approx 500 \mathrm{~K}\right)$. As shown in Fig. $2 c$, the switching of resistance state occurs when the sample chamber is pumped from argon to vacuum below $440 \mathrm{~K}$, suggesting the environmental condition plays important role for the resistivity switching. This switching feature gradually disappears as the temperature rises to the $\mathrm{CO}$ transition value $\left(\mathrm{T}_{\mathrm{co}} \approx 500 \mathrm{~K}\right)$ above which $\mathrm{LuFe}_{2} \mathrm{O}_{4}$ has a charge-disordered state with a relatively high conductivity. These facts demonstrate the presence of an essential correlation between charge ordering and gas sensitivity in present system.

In Fig. 3 the reversibility of the resistance switching in $\mathrm{LuFe}_{2} \mathrm{O}_{4}$ is discussed. We performed experimental measurements on a sample in the $\mathrm{O}_{2}$ chamber. Decrease of oxygen pressure by pumping out the $\mathrm{O}_{2}$ from the chamber to vacuum leads to a rapid resistance switch into low resistance states; then when the $\mathrm{O}_{2}$ is reintroduced into the chamber, the experimental data switches reversibly to higher resistance state. Figure 3 shows a series of the experimental results as the surrounding chemical components was cycled between vacuum $(\leq 0.1 \mathrm{~Pa})$ and $\mathrm{O}_{2}$, illustrating the remarkable resistance switching and high gas sensitivity for $\mathrm{LuFe}_{2} \mathrm{O}_{4}$ at room temperature. It is clearly recognizable that a rapid and reversible change in the resistance oscillates in response to the alternating environmental pressure. Similar resistivity switching behavior has been also observed by using the $\mathrm{LuFe}_{2} \mathrm{O}_{4}$ single crystal samples, demonstrating an essential bulk property of gas sensitivity instead of effects from surface or grain boundaries as commonly discussed for the conventional gas sensors. Besides, measurements in oxygen environment often show some additional complex changes, which are believed to be connected with the interstitial and/or deficient oxygen atoms in the layered $\mathrm{LuFe}_{2} \mathrm{O}_{4}$.

Considering the notable effects of interstitial oxygen ions on both microstructure and transport properties of $\mathrm{LuFe}_{2} \mathrm{O}_{4}$, we have also examined the changes of microstructure in association with nonlinear transition by using in situ TEM observation, which could be also important for understanding the sensing mechanism in present system. Figure 4 clearly shows the superstructure modulations in correlation with charge order and oxygen order as discussed in our previous investigations ${ }^{11}$. Following the increase of applied electric field, the superstructure spots in the diffraction pattern gradually become invisible and disappear as illustrated in Fig. 4b. Based on our TEM experimental data, we estimate that the threshold electric a
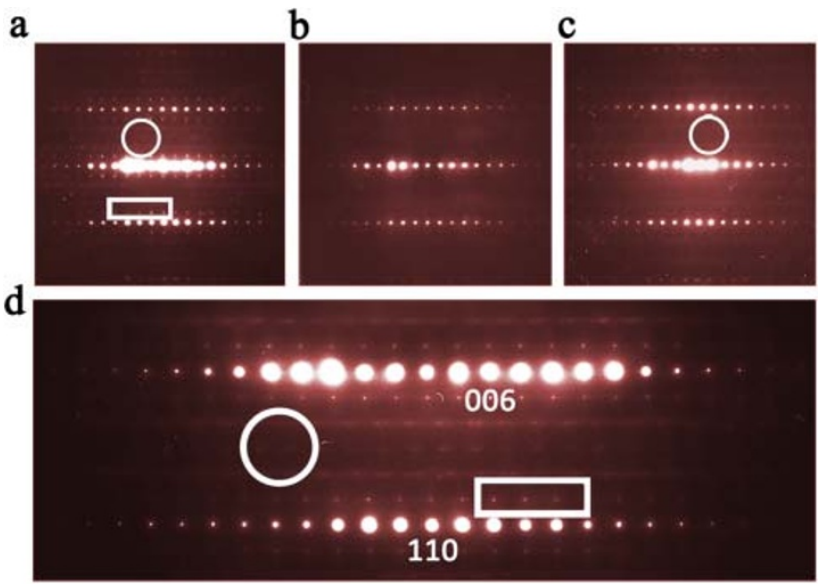

Figure $4 \mid$ The in situ TEM electron diffraction patterns taken along the [1-10] zone axis direction. (a) The diffraction spots in circle and rectangular is charge ordering and oxygen modulations respectively. (b) The charge-ordered (CO) state and oxygen modulations disappear when applied electric field is larger than $\mathrm{E}_{\mathrm{th}}(\sim 45 \mathrm{~V} / \mathrm{cm})$. (c) The CO state has recovered after the applied electric field is removed while the oxygen modulations fail to reappear. (d) The CO and oxygen modulations reemerge when the sample is placed in air for more than $24 \mathrm{hrs}$.

field is approximately $45 \mathrm{~V} / \mathrm{cm}$. After the external electric field is removed, the $\mathrm{CO}$ satellite spots in the diffraction pattern re-emerge in the same crystal but the superstructure from oxygen order shows clear irreversibility in a high-vacuum column of TEM. When the sample was placed in air for more than $24 \mathrm{hrs}$, the superstructure spots of oxygen order appear again as showed in Fig. 4 d. These facts suggest that certain microstructure features in present system also vary under the applied current, and their reversibility could also be important for technological applications of gas sensing materials.

\section{Discussion}

The gas sensing process and fundamental mechanism for the gas response in the $\mathrm{CO} \mathrm{LuFe}_{2} \mathrm{O}_{4}$ as essential issues were also concerned in our investigations. Based on localized electron variable-range hopping (VRH) picture ${ }^{12}$, the electric conductivity in the CO materials could be enormously sensitive to the modification of the

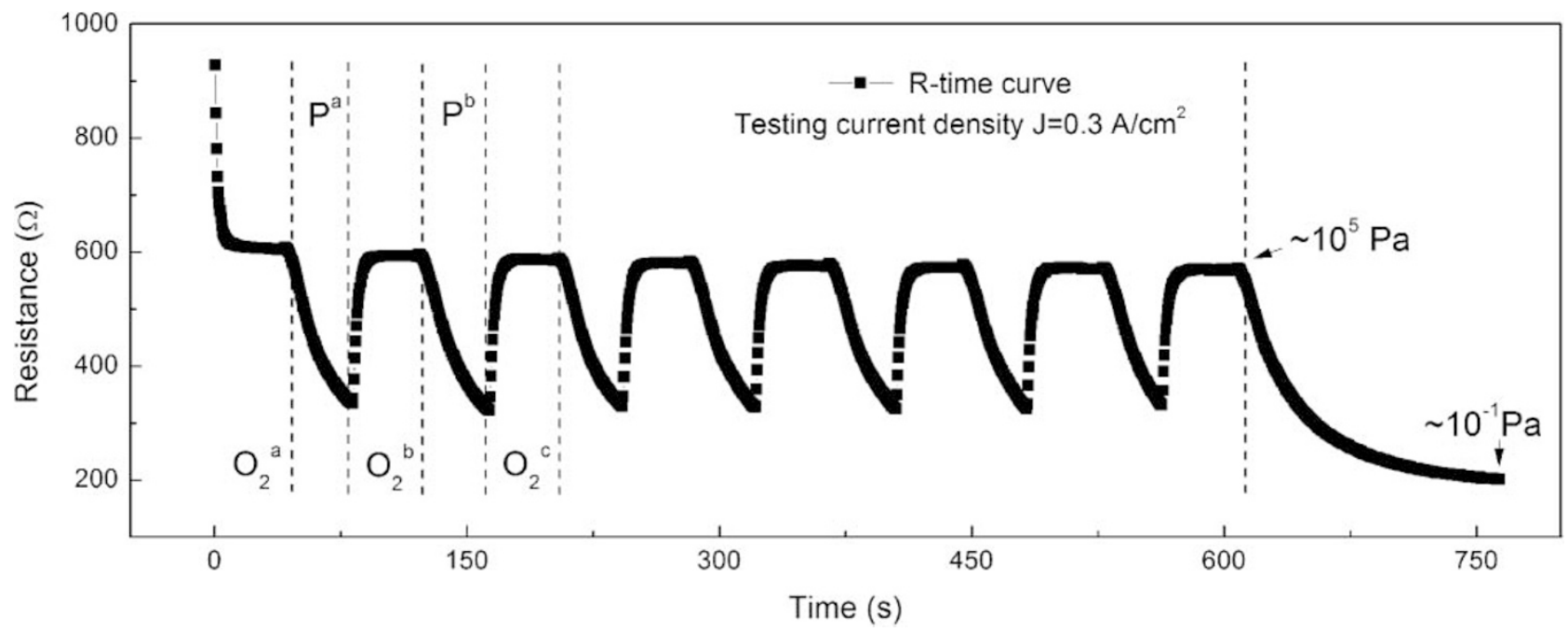

Figure $3 \mid$ Period of resistance change when switching atmosphere between oxygen and vacuum regularly. The testing current is $0.3 \mathrm{~A} / \mathrm{cm}^{2}$. In the different regions divided by dashed lines, the $\mathrm{O}_{2}{ }^{\mathrm{a}}$ is the oxygen region where sample was placed initially; $\mathrm{P}^{\mathrm{a}}$ is the region of pumping to vacuum; $\mathrm{O}_{2}{ }^{\mathrm{b}}$ is the region of introducing oxygen again; $\mathrm{P}^{\mathrm{b}}$ is the region of re-pumping vacuum. 
thermodynamic equilibrium. In a CO system, when nonlinear transition occurs above the threshold value, the melting of the $\mathrm{CO}$ state, together with the collective movement of charge stripes, can result in a strong electron-phonon interaction which contributes the Joule heating effect. Though the Joule heating could not change the essential feature of nonlinear conductivity, it indeed can induce visible alterations in the resistivity as discussed in our previous work on $\mathrm{LuFe}_{2} \mathrm{O}_{4}$ materials ${ }^{13}$. In the present study, $\mathrm{LuFe}_{2} \mathrm{O}_{4}$ was exposed to variable gaseous environments, and the thermal equilibrium states of samples depend mainly on thermal balance between Joule heating and thermal diffusion.

If the applied current density does not reach to the threshold value to trigger nonlinear conductivity, no clear gas sensitivity can be observed in our measurements. On the other hand, if the applied current is larger than the threshold value, nonlinear conductivity depends strongly on temperature and the thermal equilibrium. Because the thermal conductivity/diffusion coefficients of gases are different for $\mathrm{O}_{2}, \mathrm{Ar}$ and $\mathrm{He}$, alternation of the composition and partial pressure of gases could lead to certain changes in the thermal equilibrium, which sequentially yields a visible change in resistivity as revealed in our experiments. Under a constant applied current, it is expected that the higher conductivity/diffusion coefficient of a gas should result in a bigger resistance state, which is in a good agreement with our experimental data. For example, the conductivity coefficients for $\mathrm{He}^{14}, \mathrm{O}_{2}{ }^{15}$ and $\mathrm{Ar}^{16}$ are 156.7, 26.3 and $17.9 \mathrm{~mW} /$ $\mathrm{mK}$ at $300 \mathrm{~K}(1 \mathrm{~atm})$, respectively. The corresponding resistances are 847.7, 641.8 and $587.7 \mathrm{ohms}$ in these gas environments under a constant testing current density of $1.5 \mathrm{~A} / \mathrm{cm}^{2}$.

Careful analysis of the gas sensing process suggests a novel sensing mechanism based on the thermal conductivity/diffusion coefficients of environment gases in stark contrast with the conventional gas sensors that depend chiefly on surface reactions. Moreover, in situ TEM observation suggests that certain microstructure features in present system also are sensitive to the vacuum environment, and their reversibility could also be important for technological applications of gas sensing materials.

The experiments on other CO materials also reveal very similar sensitivity phenomena as shown in supplementary materials. These observations open up new prospects for finding new functional applications and understanding interesting physics in $\mathrm{CO}$ materials and other nonlinear materials.

\section{Methods}

The well-characterized $\mathrm{LuFe}_{2} \mathrm{O}_{4}$ polycrystal and single crystal samples used in present study were synthesized by a conventional solid-state reaction ${ }^{8}$. Polycrystalline samples of $\mathrm{LuFe}_{2} \mathrm{O}_{4}$ material were synthesized from stoichiometric mixtures of $\mathrm{Lu}_{2} \mathrm{O}_{3}$ (99.99\%) and $\mathrm{Fe}_{2} \mathrm{O}_{3}(99.99 \%)$ under a controlled oxygen partial pressure atmosphere using a $\mathrm{CO}_{2}-\mathrm{H}_{2}$ mixture at $1200^{\circ} \mathrm{C}$ for 48 hours. In order to obtain the single crystals, the polycrystalline $\mathrm{LuFe}_{2} \mathrm{O}_{4}$ power was heated up to $1620^{\circ} \mathrm{C}$ in a platinum crucible and then the melted solid was cooled to $900^{\circ} \mathrm{C}$ at a rate of $1^{\circ} \mathrm{C} / \mathrm{min}$. The typical size of the polycrystalline sample used in our measurements is $2 \times 5 \times 1 \mathrm{~mm}^{3}$. A thermocouple as commonly used in previous studies was attached on the sample holder to detect the temperature, and an additional thermometer was attached directly on the sample for certain measurements. Silver contact pads with a radius of $\sim 1 \mathrm{~mm}$ were deposited on samples. Current density-electric field (J-E) curves and transport testing were obtained by the two-probe and/or four-probe methods with a Keithley 2400/ $2611 \mathrm{~A}$ source meter. In order to protect the samples, a compliance current of $200 \mathrm{~mA}$ was used during the testing of nonlinear J-E curves. Transmission electron microscopy (TEM) investigations were performed at room temperature on a Tecnai F20
(200 kV) double-tilt TEM holder on which the in situ observations upon applied electric field can be performed.

1. Wang, C. X., Yin, L. W., Zhang, L. Y., Xiang, D. \& Gao, R. Metal Oxide Gas Sensors: Sensitivity and Influencing Factors. Sensors 10, 2088-2106 (2010).

2. Chaabouni, F., Abaab, M. \& Rezig, B. Metrological characteristics of ZNO oxygen sensor at room temperature. Sens. Actuator B-Chem. 100 (2004).

3. Collins, P. G., Bradley, K., Ishigami, M. \& Zettl, A. Extreme oxygen sensitivity of electronic properties of carbon nanotubes. Science 287, 1801-1804 (2000).

4. Tomioka, Y., Asamitsu, A., Moritomo, Y., Kuwahara, H. \& Tokura, Y. Collapse of a Charge-Ordered State under a Magnetic-Field in $\mathrm{Pr}_{1 / 2} \mathrm{Sr}_{1 / 2} \mathrm{MnO}_{3}$. Physical Review Letters 74, 5108-5111 (1995).

5. Moritomo, Y., Kuwahara, H., Tomioka, Y. \& Tokura, Y. Pressure effects on charge-ordering transitions in Perovskite manganites. Phys. Rev. B 55, 7549-7556 (1997).

6. Asamitsu, A., Tomioka, Y., Kuwahara, H. \& Tokura, Y. Current switching of resistive states in magnetoresistive manganites. Nature 388, 50-52 (1997).

7. Kiryukhin, V. et al. An X-ray-induced insulator-metal transition in a magnetoresistive manganite. Nature 386, 813-815 (1997).

8. Zhang, Y., Yang, H. X., Ma, C., Tian, H. F. \& Li, J. Q. Charge-stripe order in the electronic ferroelectric $\mathrm{LuFe}_{2} \mathrm{O}_{4}$. Physical Review Letters 98 (2007).

9. Korotcenkov, G. Metal oxides for solid-state gas sensors: What determines our choice? Mater. Sci. Eng. B-Solid State Mater. Adv. Technol. 139, 1-23 (2007).

10. Fan, Z. Y., Wang, D. W., Chang, P. C., Tseng, W. Y. \& Lu, J. G. ZnO nanowire field-effect transistor and oxygen sensing property. Appl. Phys. Lett. 85, 59235925 (2004).

11. Yang, H. X. et al. Phase separation and ferroelectric ordering in charge-frustrated $\mathrm{LuFe}_{2} \mathrm{O}_{4-\delta}$. Solid State Commun. 150, 1467-1472 (2010).

12. Mott, N. F. Conduction in non-crystalline materials. 3. localized states in a pseudogap and near extremities of conduction and valence bands. philosophical magazine 19, 835 (1969).

13. Cao, S. et al. Nonlinear transport properties and Joule heating effect in charge ordered $\mathrm{LuFe}_{2} \mathrm{O}_{4}$. Appl. Phys. Lett. 98 (2011).

14. Kestin, J. et al. Equilibrium and transport-properties of the noble-gases and their mixtures at low-density. J. Phys. Chem. Ref. Data 13, 229-303 (1984).

15. Younglove, B. A. Thermo-physical properties of fluids .1. Argon, ethylene, parahydrogen, nitrogen, nitrogen trifluoride, and oxygen. J. Phys. Chem. Ref. Data 11, 1-353 (1982).

16. Younglove, B. A. \& Hanley, H. J. M. The viscosity and thermal-conductivity coefficients of gaseous and liquid argon. J. Phys. Chem. Ref. Data 15, 1323-1337 (1986).

\section{Acknowledgements}

We would like to thank Professor Z.J. Ding for his help during manuscript preparation. This work is supported by the NSFC (Grant Nos. 90922001, 10904166, 11074292), the MOST (Grant Nos. 2011CBA00101, 2010CB923002, 2012CB821404,2006CB921301), and the Chinese Academy of Sciences.

\section{Author contributions}

J.Q.L., S.C. planned and coordinated the experiments. S.C., J.L. carried out gas sensing resistivity experiments. Z.W., S.C. performed TEM measurement and analysis. L.J.Z. prepared the figure 1. Y.B.Q. grew $\mathrm{LuFe}_{2} \mathrm{O}_{4}$ polycrystal and single crystal samples. H.F.T., C. M. and H.X.Y. helped with data interpretation. J.Q.L., S.C. wrote the paper.

\section{Additional information}

Supplementary information accompanies this paper at http://www.nature.com/ scientificreports

Competing financial interests: The authors declare no competing financial interests.

License: This work is licensed under a Creative Commons

Attribution-NonCommercial-ShareAlike 3.0 Unported License. To view a copy of this license, visit http://creativecommons.org/licenses/by-nc-sa/3.0/

How to cite this article: Cao, S. et al. Extreme chemical sensitivity of nonlinear conductivity in charge-ordered $\mathrm{LuFe}_{2} \mathrm{O}_{4}$. Sci. Rep. 2, 330; DOI:10.1038/srep00330 (2012). 